\title{
Effect of Injected Charges in Polydimethylsiloxane Films on Triboelectric Power Generation
}

\author{
Ryuto Takita, ${ }^{1}$ Kaito Koshiji, ${ }^{1}$ Hiroki Kokubo, ${ }^{1}$ \\ Winadda Wongwiriyapan, ${ }^{2}$ and Takashi Ikuno ${ }^{1 *}$ \\ ${ }^{1}$ Graduate School of Advanced Engineering, Department of Applied Electronics, \\ Tokyo University of Science, Katsushika, Tokyo 125-8585, Japan \\ ${ }^{2}$ College of Nanotechnology, King Mongkut's Institute of Technology Ladkrabang, Bangkok 10520, Thailand
}

(Received May 19, 2021; accepted September 6, 2021)

Keywords: triboelectricity, electret, corona discharge, polydimethylsiloxane

Towards the application of stand-alone power supplies for wearable sensors, we have investigated the effect of fixed charges in a dielectric polymer film of a triboelectric device on the output power. Our device is composed of an aluminum (Al) plate and a polydimethylsiloxane (PDMS) film. Using a corona discharge gun, we injected negative charges into the PDMS film. To maximize the output power, we optimized the corona discharge conditions. The output voltage and output power of the device using the charged PDMS film fabricated under the optimum conditions were 4.5 and 20 times higher than those of the device using the pristine PDMS film, respectively.

\section{Introduction}

Triboelectric devices, which can generate electric power by contact and/or friction between different objects, ${ }^{(1-6)}$ are attracting attention as a power source for wearable devices and small sensors $^{(7)}$ because of their simple structure and compact size. ${ }^{(8,9)}$ However, the output power of triboelectric devices is lower than that of other power generation devices. ${ }^{(10)}$ Researchers are eager to improve the output power by optimizing the combination of materials, increasing the effective surface area, and modifying the surface. ${ }^{(1-13)}$ For example, although a previously reported triboelectric device composed of stacked layers of polyethylene terephthalate (PET) and polyimide was simple and inexpensive, it showed a low output voltage of $3.3 \mathrm{~V}$ (maximum power density of $\left.0.367 \mu \mathrm{W} / \mathrm{cm}^{2}\right){ }^{(11)}$ A device composed of a combination of a pyramid-patterned polydimethylsiloxane (PDMS) film and a transparent conductive oxide/PET film was reported to improve output power. It achieved an output voltage of $18 \mathrm{~V}$, a current of $0.7 \mu \mathrm{A}$, and a power density of $2.34 \mu \mathrm{W} / \mathrm{cm}^{2}{ }^{2}{ }^{(12)}$ Another device composed of a patterned PDMS film and an Al plate in the shape of an arch provided a high output voltage of $230 \mathrm{~V}$, a high output current of $0.13 \mathrm{~mA}$, and a power density of $3.56 \mathrm{~mW} / \mathrm{cm}^{2}{ }^{2}{ }^{(13)}$ Although these patterned polymer and arch devices provided high output power, they are expected to have a high processing cost.

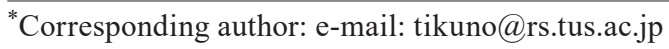

https://doi.org/10.18494/SAM.2021.3434 
In this study, we aimed to realize a triboelectric device with high output power using a simple structure and materials. We used a polymer film with a fixed charge formed beforehand and obtained the relationship between the power generation characteristics and the parameters involved in the formation of the fixed charge in the film.

\section{Experimental Procedure}

Two different materials, such as a polymer film and metal plate, are required to generate electric power from triboelectric devices. We adopted a PDMS film and an aluminum (Al) plate as the materials in this study. We used PDMS because it not only has various functions such as heat resistance, low surface tension, and water repellency, but it is also easily negatively charged. ${ }^{(14,15)}$ Since the aim of this study was to increase the output power by forming fixed negative charges in the polymer film, PDMS is an appropriate electrode material.

SYLGARD184 (Dow Corning) PDMS films were used in this study. The base and agent of the product were mixed in a mass ratio of 10:1. We removed bubbles generated during mixing with a vacuum pump. The mixture was then poured into a mold and heated at $100{ }^{\circ} \mathrm{C}$ for $35 \mathrm{~min}$ to harden it. The lateral size of the obtained PDMS film was $6 \mathrm{~cm} \times 3 \mathrm{~cm}$ and the thickness was approximately $0.8 \mathrm{~mm}$. An Al plate (M9501, Kuho Co. Ltd.) was placed against the PDMS film as a counter electrode, which plays the role of forming a contact voltage and extracting charge.

After preparing the PDMS film and the Al plate, negative charges were injected into the PDMS film using a corona discharge gun (GC90N, Green Techno) to increase the output power. Figure 1 schematically shows the experimental setup for charge injection. A PDMS film was placed on a grounded Al plate substrate and heated to $60-200{ }^{\circ} \mathrm{C}$ using a hot plate, then a discharge voltage $V_{d}$ was applied between the corona discharge gun and the $\mathrm{Al}$ substrate. The distance between the gun and the substrate was fixed as $d$. The corona discharge ionized the gas molecules in the air and injected an electric charge into the PDMS film. ${ }^{(16)}$

As shown in Fig. 2(a), when the PDMS film and the Al counter electrode are in contact, negative and positive charges are induced on the top and bottom surfaces of the PDMS film, respectively. At this time, a current flows to the external circuit. Next, when the PDMS film and

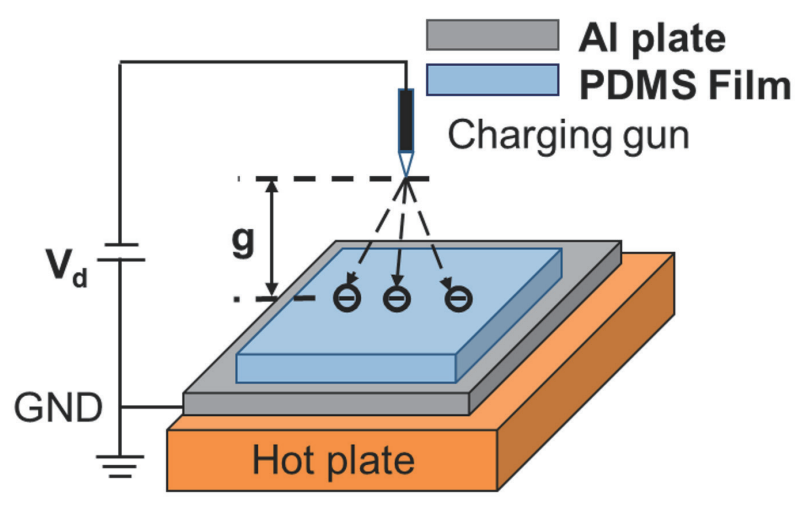

Fig. 1. (Color online) Schematic illustration of experimental setup of charge injection. 
(a)

Contact

Separation

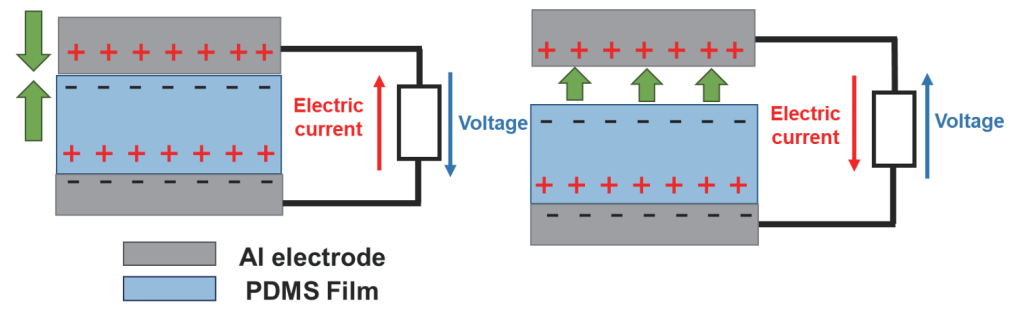

(b)

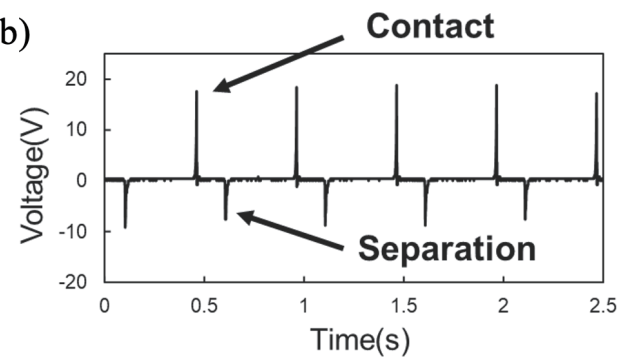

Fig. 2. (Color online) (a) Measurement circuit and (b) output voltage waveform.

the $\mathrm{Al}$ counter electrode are separated, the current flows to the external circuit in the opposite direction from that described above. In this study, we used a $1 \mathrm{M} \Omega$ resistor as the external load and measured the electromotive force generated in the resistor. As a result, we obtained the waveform shown in Fig. 2(b). We obtained the output voltage from the peak-to-peak voltage $V_{p-p}$ of the waveform. We typically adopted a contact/separation frequency of $2 \mathrm{~Hz}$.

\section{Results and Discussion}

We measured the output voltage under different discharge conditions, i.e., the annealing temperature during injection $T_{S}$, the corona discharge voltage $V_{d}$, and the distance between the gun and the PDMS film $g$.

First, the dependence of the output voltage on the annealing temperature is shown in Fig. 3. Here, the PDMS films were fabricated with a constant discharge voltage $\left(V_{d}=-40 \mathrm{kV}\right)$, a constant distance $g$ of $5 \mathrm{~cm}$, and different annealing temperatures. An output voltage, namely $V_{p-p}$ of the waveform, of approximately $13.2 \mathrm{~V}$ was obtained for the unheated sample. For the annealed samples, an almost constant output voltage of about $19.2 \pm 2.5 \mathrm{~V}$ was obtained regardless of the annealing temperature.

Next, the dependence on the corona discharge voltage was investigated, where the annealing temperature was $80{ }^{\circ} \mathrm{C}$ and the gap was $5 \mathrm{~cm}$ during the fabrication of the PDMS films. Figure 4(a) shows the time evolution of the output voltage for each corona discharge voltage. It was found that the output voltage increased with increasing discharge voltage. For the discharge voltage of $-40 \mathrm{kV}$, the output voltage under contact was approximately $20 \mathrm{~V}$, the voltage under separation was approximately $12 \mathrm{~V}$, and $V_{p-p}$ was approximately $32 \mathrm{~V}$. As shown in Fig. 4(b), the output voltage was proportional to the corona discharge voltage. 


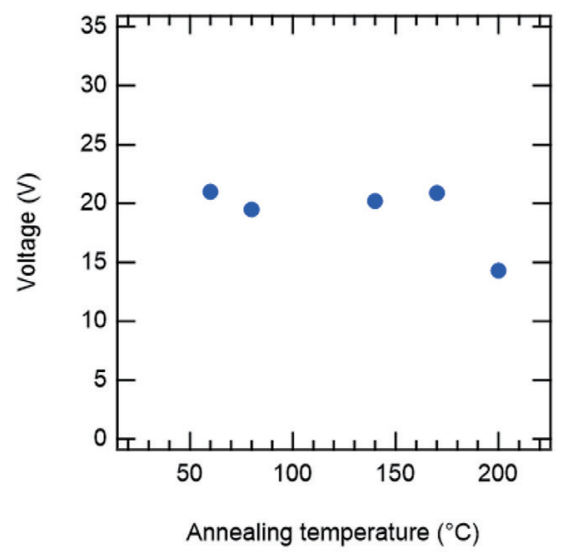

Fig. 3. (Color online) Output voltage as a function of annealing temperature.

(a)

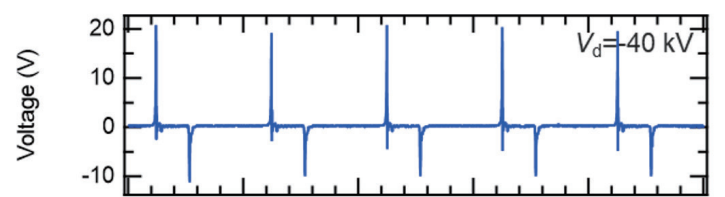

(b)
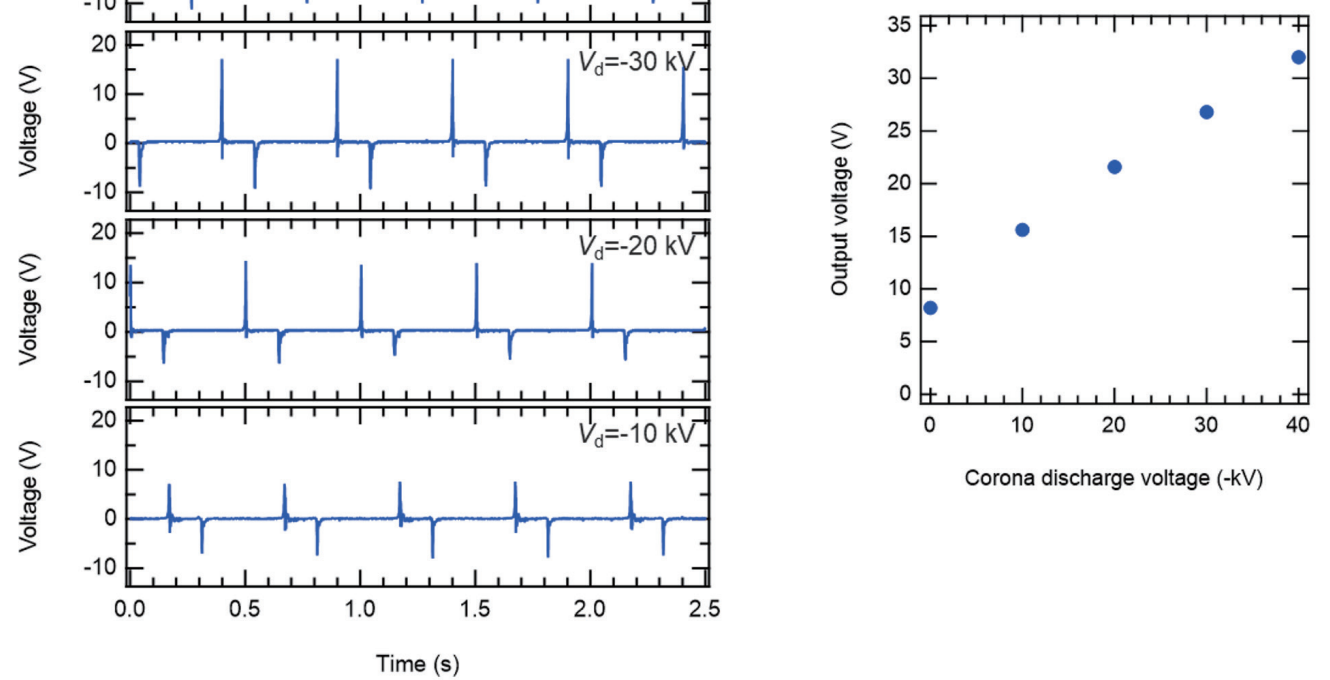

Fig. 4. (Color online) (a) Time evolution of output voltage for each corona discharge voltage and (b) output voltage as a function of corona discharge voltage.

In general, $V_{s}$ is proportional to the surface charge density $\sigma$ of a PDMS film ${ }^{(17)}$ as follows:

$$
\sigma=\varepsilon_{s} \cdot \varepsilon_{0} \frac{V_{s}}{d}
$$

where $V_{s}$ is the output voltage, $\varepsilon_{s}$ is the relative permittivity of PDMS, $\varepsilon_{0}$ is the vacuum permittivity, and $d$ is the thickness of the PDMS film. The data points in Fig. 4(b) could be fitted 
with a straight line with a slope of $0.59 \mathrm{~V} / \mathrm{kV}$. In other words, the higher the corona discharge voltage, the more charges could be injected into the PDMS surface, resulting in a higher output voltage. Moreover, the surface charge density increased linearly with the corona discharge voltage.

Finally, we investigated the relationship between the gap $g$ and the output voltage. Figure 5(a) shows the time variation of the output voltage for each gap between the corona gun and the PDMS film. Although $V_{p-p}$ appears to increase as the gap increases, the output voltage at $g=5 \mathrm{~cm}$ was smaller than that at $g=4 \mathrm{~cm}$. Figure 5(b) shows the relationship between the output voltage and the gap. The output voltage increased with the gap up to $4 \mathrm{~cm}$ and then decreased. In other words, there is an optimum gap for obtaining a high output voltage, which was found to be approximately $4 \mathrm{~cm}$.

Since the corona discharge is expected to irradiate the ion flux to the PDMS radially, the area of the PDMS surface that is irradiated depends on the gap. The reason why the output voltage decreased when the gap was larger than $4 \mathrm{~cm}$ was because the irradiated area exceeded the area of the PDMS.

From the above results, an appropriate annealing temperature is 60 to $170{ }^{\circ} \mathrm{C}$, the optimum corona discharge power is $-40 \mathrm{kV}$, and the optimum distance between the gun and the film is $4 \mathrm{~cm}$ for our PDMS film. The output voltage and output power of the device fabricated under the optimum conditions were 4.5 and 20 times higher than those of the device using the pristine PDMS film, respectively. In this experiment, we were able to increase the voltage by injecting a

(a)

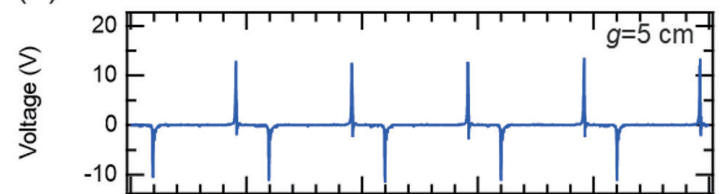

(b)
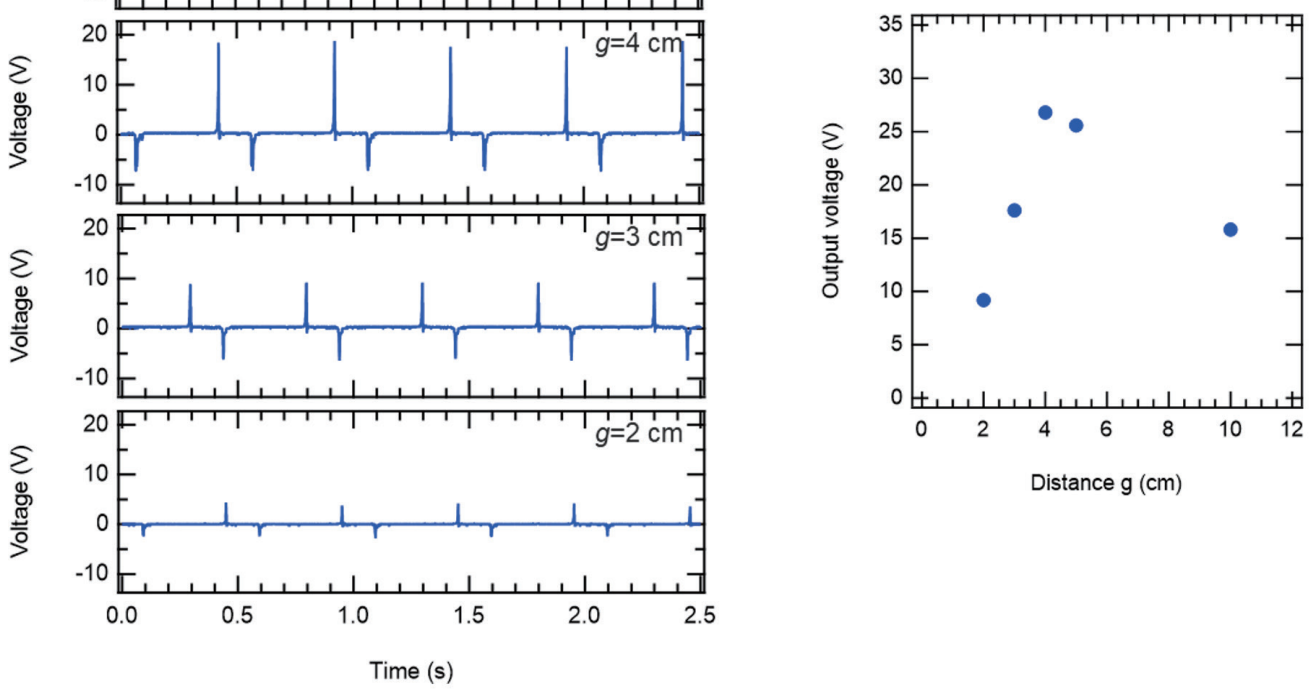

Fig. 5. (Color online) (a) Time evolution of output voltage for each gap between corona gun and PDMS film and (b) output voltage as a function of gap. 
charge into the PDMS, but the voltage generated can also be increased by increasing the speed of the contact and separation.

Although not mentioned above, we also found that a voltage of $64.8 \mathrm{~V}$ or higher can be obtained by tapping the device by hand with nitrile gloves. As a demonstration, we connected the triboelectric device to an LED, which was the load. When the triboelectric device was tapped by hand, the connected LED emitted light intermittently.

\section{Conclusions}

In this study, the output voltage of a triboelectric generator composed of a PDMS film and an Al plate was increased by forming a fixed charge on the PDMS. After optimizing the conditions during charge injection, an appropriate annealing temperature was found to be $60-170{ }^{\circ} \mathrm{C}$, the optimum corona discharge voltage was $-40 \mathrm{kV}$, and the optimum distance between the gun and the film was $4 \mathrm{~cm}$. The output voltage and output power of the device using the electret PDMS film fabricated under the optimized conditions were 4.5 and 20 times higher than those of the device using the pristine PDMS film, respectively. We believe that this method is applicable to not only PDMS but also other materials such as polyimide and PET. In the future, we will utilize the flexible triboelectric generator developed in this study as a power source for wearable sensors.

\section{References}

1 G. Zhu, Z. H. Lin, Q. Jing, P. Bai, C. Pan, Y. Yang, Y. Zhou, and Z. L. Wang: Nano Lett. 13 (2013) 2. https://doi. org $/ 10.1021 / \mathrm{n} 14001053$

2 S. Niu. S. Wang, L. Lin, Y. Liu, Y. S. Zhou, Y. Hu, and Z. L.Wang: Energy Environ. Sci. 6 (2013) 3576. https:// doi.org/10.1039/C3EE42571A

3 R. G. Horn, D. T. Smith, and A. Grabbe: Nature 366 (1993) 442. https://doi.org/10.1038/366442a0

4 R. G. Horn and D. T. Smith: Science 256 (1992) 362. https://doi.org/10.1126/science.256.5055.362

5 L. S. McCarty and G. M. Whitesides: Angew. Chem. Int. Ed. 47 (2008) 2188. https://doi.org/10.1002/ anie. 200701812

6 H. T. Baytekin, A. Z. Patashinski, M. Branicki, B. Baytekin, S. Soh, and B. A. Grzybowski: Science 333 (2011) 308. https://doi.org/10.1126/science.1201512

7 Z. L. Wang: Faraday Discuss. 176 (2014) 447. https://doi.org/10.1039/C4FD00159A

8 G. Zhu, J. Chen, T. Zhang, Q. Jing, and Z. L. Wang: Nat. Commun. 5 (2014) 3426. https://doi.org/10.1038/ ncomms 4426

9 G. Zhu, C.Pan, W. Guo, C. Chen, Y. Zhou, R. Yu, and Z. L. Wang: Nano Lett. 12 (2012) 9. https://doi. org $/ 10.1021 / \mathrm{n} 1302560 \mathrm{k}$

10 Z. L. Wang: ACS Nano 7 (2013) 11. https://doi.org/10.1021/nn404614z

11 F. R. Fan, Z. Tian, and Z. L. Wang: Nano Energy 1 (2012) 2. https://doi.org/10.1016/j.nanoen.2012.01.004

12 F. R. Fan, G. Zhu, W. Wu, R. Zhang, and Z. L. Wang: Nano Lett. 6 (2012) 12. https://doi.org/10.1021/nl300988z

13 S. Wang, L. Lin, and Z. L. Wang: Nano Lett. 12 (2012) 12. https://doi.org/10.1021/nl303573d

14 I. Firdous, M. Fahim, and W. A. Daoud: Nano Energy 82 (2021) 105694. https://doi.org/10.1016/j. nanoen.2020.105694

15 Y. Yang, H. Zhang, Z. Lin, Y. S. Zhou, Q. Jing, Y. Su, J. Yang, J. Chen, C. Hu, and Z. L. Wang: ACS Nano 7 (2013) 10. https://doi.org/10.1021/nn403838y

16 M. A. Eddings, M. A. Johnson, and B. K. Gale: J. Micromech. Microeng. 18 (2008) 6. https://doi. org/10.1088/0960-1317/18/6/067001

17 Z. L. Wang: Adv. Energy Mater. 10 (2020) 17. https://doi.org/10.1002/aenm.202000137 


\section{About the Authors}

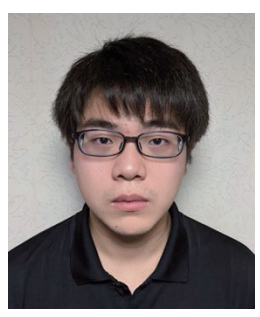

Ryuto Takita was born in Chiba, Japan, in 1998. He received his bachelor's degree from Tokyo University of Science in 2021. Currently, he is a student at Tokyo University of Science majoring in electronic systems engineering. He is studying energy conversion devices. (8121525@ed.tus.ac.jp)

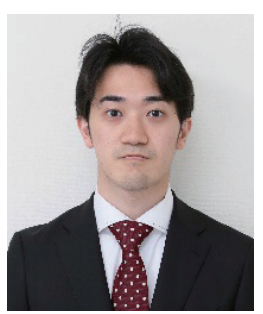

Kaito Koshiji was born in Tokyo, Japan, in 1997. He received his bachelor's degree from Tokyo University of Science in 2020. Currently, he is a student at Tokyo University of Science majoring in electronic systems engineering. He is studying energy conversion devices. (8120524@ed.tus.ac.jp)

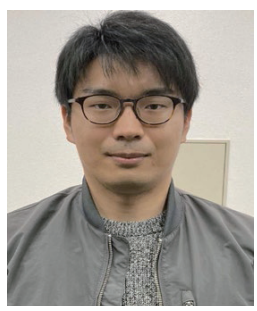

Hiroki Kokubo was born in Aichi, Japan, in 1995. He received his master's degree in engineering from Tokyo University of Science in 2021. His area of research is energy conversion devices. (hkokubo@ikunolab.net)

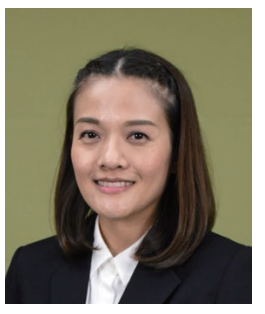

Winadda Wongwiriyapan is an associate professor of the College of Nanotechnology, King Mongkut's Institute of Technology Ladkrabang, Thailand. She received her Ph.D. degree from Osaka University, Japan, in 2008. She also received postdoctoral fellowships from the Institute of Carbon Science and Technology, Shinshu University, Japan, and Sungkyukwan University, Republic of Korea. Her research interests are the synthesis of carbon nanomaterials and their applications in sensor and energy storage devices. (winadda.wo@kmitl.ac.th)

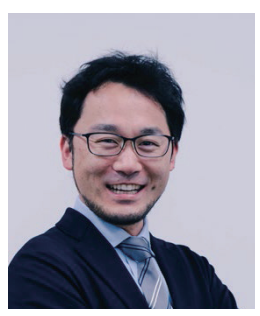

Takashi Ikuno received his Ph.D. degree from Osaka University, Japan, in 2004. From 2004 to 2007, he was a postdoctoral researcher at the University of California, Berkeley, and Lawrence Berkeley National Laboratory. From 2007 to 2016, he was a senior researcher at Toyota Central R\&D Labs. Since 2016, he has been an associate professor at Tokyo University of Science. His research interests are in carbon-related nanomaterials, energy conversion devices, and environmentally friendly materials and devices.

(tikuno@rs.tus.ac.jp) 